\title{
Optimal Planning of Communication System of CPS for Distribution Network
}

\author{
Ting Yang, Zhiyong Huang, Haibo Pen, and Yajian Zhang \\ Key Laboratory of Smart Grid, Ministry of Education, Tianjin University, Tianjin, China \\ Correspondence should be addressed to Ting Yang; yangting@tju.edu.cn
}

Received 4 November 2016; Revised 27 December 2016; Accepted 16 February 2017; Published 7 March 2017

Academic Editor: Jose N. De Souza

Copyright (C) 2017 Ting Yang et al. This is an open access article distributed under the Creative Commons Attribution License, which permits unrestricted use, distribution, and reproduction in any medium, provided the original work is properly cited.

IoT is the technical basis to realize the CPS (Cyber Physical System) for distribution networks, with which the complex system becomes more intelligent and controllable. Because of the multihop and self-organization characteristics, the large-scale heterogeneous CPS network becomes more difficult to plan. Using topological potential theory, one of typical big data analysis technologies, this paper proposed a novel optimal CPS planning model. Topological potential equalization is considered as the optimization objective function in heterogeneous CPS network with the constraints of communication requirements, physical infrastructures, and network reliability. An improved binary particle swarm optimization algorithm is proposed to solve this complex optimal problem. Two IEEE classic examples are adopted in the simulation, and the results show that, compared with benchmark algorithms, our proposed method can provide an effective topology optimization scheme to improve the network reliability and transmitting performance.

\section{Introduction}

With the development of network technology, computer technology, and embedded technology, the National Natural Science Foundation of the United States proposed the Cyber Physical Systems (CPS), which is a kind of novel intelligent complex systems tightly integrated and interacted with different scales of computation, communication, and physical components in future networks $[1,2]$. As a typical application domain, CPS's application in the power distribution network can integrate and optimize various distributed renewable energy resources, called CPSDN, and make the complex electrical power system become more intelligent and controllable [3].

Wireless sensor technology and IoT (Internet of Things) as two core technologies for CPSDN [4] make its components become heterogeneous. A CPSDN usually contains master station, substation units, CPS terminals, and physical connection lines [5]. CPS terminals can be further classified as feeder terminal, distribution terminal, distributed energy terminal, and so forth. Each terminal has its specific service object like relay protection, distribution automation, business electricity information collection, distributed cooperative control, production management, environmental monitoring, and so forth. In order to meet the requirements in CPSDN such as overall perception and real-time control, an optimal CPS network infrastructure planning to integrate those heterogeneous components is very important, but it is also more difficult.

In previous research on distributed network planning, scholars paid more attention to homogeneous system, such as homogeneous wireless sensor network, and the energyefficient is the primary aim $[6,7]$. Liu et al. proposed the fault tolerant topology evolution model with energy consumption and load in wireless sensor networks [8] which can simultaneously balance the network node energy consumption and the lifetime extension of the network. In addition, an evolutionary multiobjective optimization approach based on nondominated sorting genetic algorithm was proposed in [9] to simultaneously optimize balanced energy consumption, maximize covered area, minimize the number of active nodes and maintain the connectivity of active nodes, and then improve the performance of WSN. However, the above researches ignored the impact of heterogeneous 
TABLE 1: Typical acquisition points and communications requirement of CPSDN terminal units.

\begin{tabular}{lccccc}
\hline Unit & $\begin{array}{c}\text { Remote } \\
\text { signal }\end{array}$ & $\begin{array}{c}\text { Number of pieces of information } \\
\text { Remote } \\
\text { measurement }\end{array}$ & $\begin{array}{c}\text { Remote } \\
\text { control }\end{array}$ & Delay & Traffic flow \\
\hline FTU & 13 & 11 & 2 & $<500 \mathrm{~ms}$ & $12 \mathrm{bytes} / \mathrm{s}$ \\
TTU & 15 & 14 & 6 & $<3 \mathrm{~s}$ & $23 \mathrm{bytes} / \mathrm{s}$ \\
\hline
\end{tabular}

node communication capability on the network topology construction. Considering the heterogeneity of sensor nodes' communication capabilities, Sun et al. proposed an area energy consumption rate function which was used to estimate the energy consumption rate of communication areas and determine the selection of dominating nodes by integrating the quality of communication links, transmission range, and remaining energy of nodes [10]. Hu et al. modeled the distribution network and its communication subnetwork based on complex network theory and presented a topology optimization strategy to improve network's static and dynamic reliability [11]. Different from the traditional wireless sensor network, CPS should consider not only the communication requirements, but also the heterogeneous demands from the service objects of physical system. Since the reliable energy supply and complete UPS mechanisms, the primary parameters in CPSDN planning are network reliability and quality of service instead of energy consumption.

This paper fully considers the heterogeneous characteristics of CPSDN and proposes a novel topology potential equilibrium model to handle CSPDN planning problem. By defining the generalized node quality for the heterogeneous CPS terminals, the mutual influence of nodes and the spatial distribution of topological structure can be mathematically described. An improved binary particle swarm optimization algorithm is used to solve this complex optimal problem. Two IEEE classic examples, IEEE 39-bus and IEEE LVNTS, are adopted in the simulation to evaluate the network reliability and transmitting performance with proposed algorithm.

The organizational structure of this paper is as follows. Section 1 reviews related work in literatures, followed by Section 2 identifying the heterogeneous characteristics of CPSDN. The generalized node quality and the topological potential equilibrium CPSDN planning model are also proposed in Section 2. Section 3 presents an improved binary particle swarm optimization with adaptive weights to solve the model. In Section 4, the simulation results are analyzed with IEEE 39-bus and IEEE LVNTS to evaluate performance of proposed algorithm. The conclusion remarks and future work are given in Section 5 finally.

\section{Topological Potential Equilibrium CPSDN Planning Model}

Different from the homogeneous nodes in wireless sensor network or IoT, the CPS terminals in power distribution network are heterogeneous from communication requirement and information models. Various demands of the service objects in physical system make the CPSDN planning problem as a complex optimal problem. With predefined generalized node quality, the CPSDN planning problem can be mathematically modeled by topological potential equilibrium. Firstly, the heterogeneous characteristics of CPSDN terminals are discussed from both cyber and power physical systems.

2.1. Multisource Heterogeneous Characteristics in CPSDN. In CPSDN planning, the heterogeneity and interaction of CPS terminals should be considered. Obviously, the CPS service object is heterogeneous electrical equipment [12]. Moreover, CPSDN terminals connected with corresponding electrical equipment also collect different types and quantity of data [13]. Consequently, the computing capabilities and communication requirements are different. Table 1 presents the typical acquisition points $[14,15]$ and communication requirements of CPSDN terminal units. The structures of feeder terminal unit (FTU) and transformer terminal unit (TTU) [16] are presented in Figure 1. Therefore, it is necessary to establish a unified CPSDN planning model to describe the heterogeneity.

\subsection{Problem Statement with Topological Potential Theory}

Theorem 1. Assume that the data object $j$ is in the given space $\Omega$, and $\vee j \in \Omega$. The potential value $\varphi_{j}(i)$ is generated by the object $j$ at the point $i$. And $\varphi_{j}(i)$ should meet the following conditions at the same time [17]:

(1) $\varphi_{j}(i)$ is a smooth, continuous, and finite function which is defined in space $\Omega$.

(2) $\varphi_{j}(i)$ is isotropic.

(3) $\varphi_{j}(i)$ is a monotone increasing function of the distance $\|i-j\|$. When the distance $\|i-j\|$ equals zero, $\varphi_{j}(i)$ reaches its peak which is not infinite. And when $\|i-j\|$ tends to infinity, $\varphi_{j}(i)$ tends to 0 .

The potential function [18] can be defined by the above criteria:

$$
\varphi_{j}(i)=m \times e^{-(\|i-j\| / \sigma)^{k}},
$$

where $\varphi_{j}(i)$ represents the potential value generated by the object $j$ at the point $i$ and $m$ is the quality of data objects $j$. $\|i-j\|$ is the distance between the object $j$ and the point $i$. In addition, $\sigma$ is used to control the interactive force among objects and $k$ represents the distance index. When $k=2, \varphi_{j}(i)$ is called the Gauss potential [19].

This theorem illustrates how to establish topological potential function. The topological potential theory can 


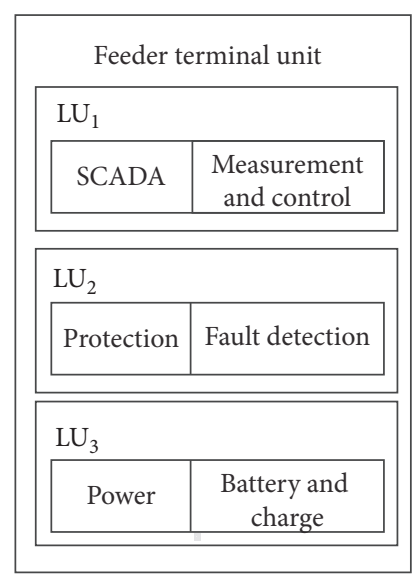

(a)

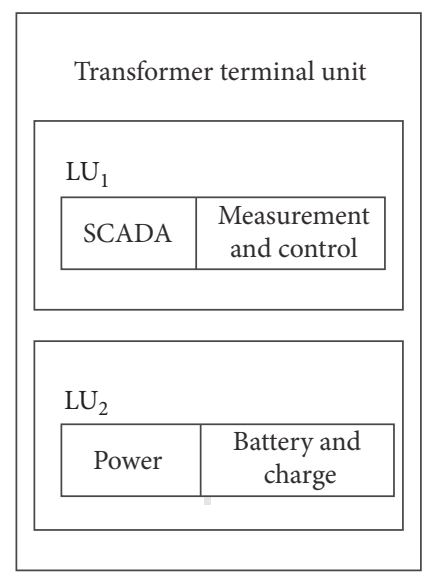

(b)

FIgURE 1: The structure of feeder terminal unit and transformer terminal unit.

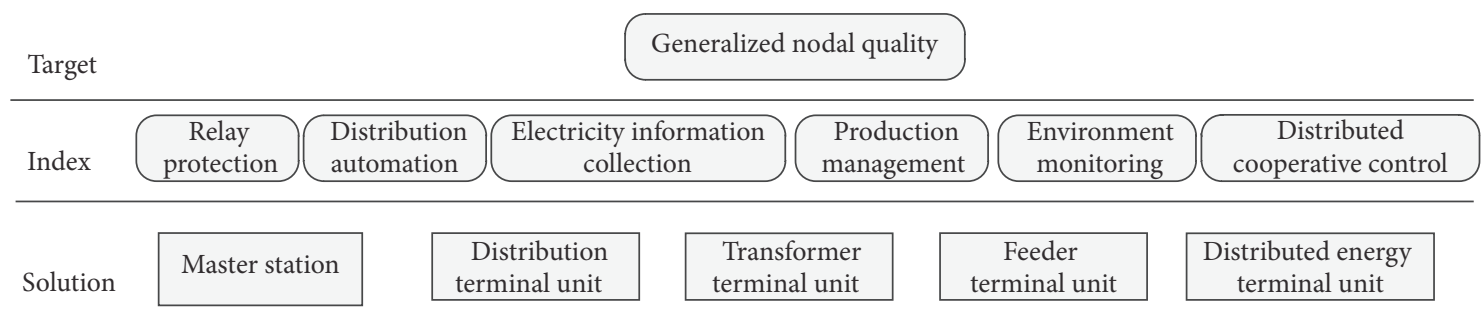

FIGURE 2: Hierarchical structure of generalized quality.

accurately represent the weight of each node in networks. Distributing the topological potential equally will improve the reliability of the network to resist the attack or units' fault. Therefore, this paper defines the CPSDN generalized node quality and then establishes the topological potential equilibrium CPSDN planning model.

\subsection{Generalized Quality and Potential Function of CPSDN}

2.3.1. Definition of Generalized Node Quality. Based on above all, weight of nodes is different for its multisource heterogeneous characteristic of CPSDN. In order to assess the weight of nodes, we define the generalized node quality. The numerical results are obtained by analytic hierarchy process (AHP).

(1) Hierarchical Structure of Generalized Node Quality. The services of CPSDN mainly contain relay protection, power distribution automation, electrical information collection, distributed cooperative control, production management, environmental monitoring, and so forth. This paper classifies the CPSDN units into masters and terminals. Moreover, the latter can be categorized into feeder terminals, distribution terminals, transformer terminals, and distributed energy terminals according to installation locations. As shown in
Figure 2, three layers are set to calculate the generalized node quality.

(2) Generalized Node Quality Calculating of CPSDN Terminals. The generalized quality of communication nodes can be obtained by the largest eigenvalues of judgment matrixes in each hierarchy, namely,

$$
m_{k}=\sum_{l=1}^{n} \omega_{l k} W_{l} \text {, }
$$

where $m_{k}$ is the generalized quality of $k$ th node; $\left[\omega_{l 1}, \omega_{l 2}, \ldots\right.$, $\left.\omega_{l k}, \ldots, \omega_{l m}\right]^{T}$ is the eigenvector corresponding to the largest eigenvalue of the judgment matrix belonging to $l$ th index which is calculated by solution layer; $\left[W_{1}, W_{2}\right.$, $\left.\ldots, W_{l}, \ldots, W_{n}\right]^{T}$ is the eigenvector corresponding to the largest eigenvalue of the judgment matrix which is calculated by index layer. The judgment matrix $A$ can be calculated as

$$
A=\left[a_{i j}\right]_{n \times n},
$$

where $a_{i j}$, the diagonal symmetry elements, is the importance ratio between two CPSDN terminals $i$ and $j . a_{i j}$ and $a_{j i}$ meet reciprocal relationship [20]. 
2.3.2. Definition of Potential Function. To evaluate the reliability of network, the potential function of CPSDN is defined as follows:

$$
\begin{aligned}
& \varphi(i)=\sum_{j=1}^{n} \varphi_{j}(i)=\sum_{j=1}^{n}\left(m_{j} \times e^{-(\|i-j\| / \sigma)^{2}}\right), \\
& \varphi_{j}(i)=m_{j} \times e^{-(\|i-j\| / \sigma)^{k}},
\end{aligned}
$$

where $\varphi_{j}(i)$ is the algebraic sum of potential in ith communication node produced by all the communication nodes (including $i$ itself); $\varphi_{j}(i)$ is the potential of $i$ th communication node produced by $j$ th communication node; $m_{j}$ is the generalized quality of $j$ th communication node; $\|i-j\|$ is the shortest path hop between $i$ th and $j$ th nodes. $\sigma$ is the interactive force index between communication nodes. $k$ is the distance index.

\subsection{Objective Function and Constraints of CPSDN Optimal} Planning. To improve the reliability of CPSDN, the nodal potential should tend to equilibrium. For the more the equilibrium among each CPSDN terminal's potential, the more the reliability topology. Mean square error (MSE) of potential is chosen as the objective function in CPSDN planning, expressed as follows:

$$
\min F(G)=\sum_{i=1}^{n} \frac{1}{n}\left(\varphi_{i}-\bar{\varphi}\right)^{2} .
$$

There are three constraints: the first one is that each CPSDN terminal should have at least two connected links to ensure network's connectivity, $\operatorname{Degree}(i) \geq 2$; the second one is service constraints; the third one is the maximum communication distance between two communication terminals, $l_{i j}<$ Dist $_{\max }$.

\section{Binary Particle Swarm Optimization Algorithm}

The distributed network planning problem with multiconstraints is proved as NP-complex problem [21]. It is hard to reach the global optimal results in limited time with increasing network scale. Hence, an improved binary particle swarm optimization (BPSO) algorithm is proposed in this paper to solve this problem.

In the improved binary particle swarm optimization algorithm, the position encoding of the particles is in the binary mode, where each dimension component of the particle position is limited to 0 or $1 ; 1$ means that the location of the corresponding communication link exists. The velocity of the particle is considered as the probability of the position change in this paper and bounded by the sigma function to the interval $[0,1][22]$.

In the algorithm, $N, D$, and $M$ are population size, decision space, and maximum number of iterations, respectively. The coordinate position of particle $i$ at time $d$ is expressed as $x_{i d}$; velocity of particle is defined as the distance of the particle moves in each iteration which is expressed as $v_{i d}$. Hence, the
TABLE 2: The partition of communication nodes.

\begin{tabular}{lcc}
\hline Number & Node types & Node label \\
\hline 1 & Master station & 8 \\
2 & Distribution terminal unit & $1,2,3,9,13,15,16$ \\
3 & Transformer terminal unit & $5,6,7,10,11,12,14$ \\
4 & Feeder terminal unit & - \\
5 & Distributed energy unit & 4,17 \\
\hline
\end{tabular}

flight speed and position of particle $i$ at time $d$ are adjusted according to the following formula:

$$
\begin{aligned}
& v_{i d}=\omega_{d} \cdot v_{i d}+\varphi_{1} \cdot\left(p_{i d}-x_{i d}\right)+\varphi_{2} \cdot\left(p_{g d}-x_{i d}\right), \\
& x_{i d}= \begin{cases}1 & \text { if rand }()<\operatorname{sig}\left(v_{i d}\right) \\
0 & \text { else, }\end{cases}
\end{aligned}
$$

where $\omega_{d}$ is the inertia weight of the current time, $\varphi_{1}$ and $\varphi_{2}$ are acceleration factors, and $p_{i d}$ and $p_{g d}$ are the optimal position of the particles and the history of the entire group, respectively. Function $\operatorname{sig}\left(v_{i d}\right)=1 /\left(1+\exp \left(-v_{i d}\right)\right)$ is a conversion limit function to ensure that each component is limited to $[0,1]$ interval.

The adaptive weight is calculated according to the following formula [23], which can ensure the global optimization and local convergence:

$$
\omega_{d}=\omega_{\min }+\frac{\left(f v(i)-f_{\min }\right) *\left(\omega_{\max }-\omega_{\min }\right)}{\left(f_{\mathrm{ag}}-f_{\min }\right)}
$$

where $\omega_{\min }$ and $\omega_{\max }$ are the minimum and maximum value of inertia weight. $f v(i)$ is the fitness function value of particle i. $f_{\text {ag }}$ is the average of fitness function with $N$ particles. $f_{\min }$ and $f_{\max }$ are the minimum and maximum values of $N$ fitness function values, respectively.

The complete steps of the algorithm are expressed as in Algorithm 1.

\section{Simulation and Performance Evaluation}

We perform a range of simulation experiments to test the performances of the novel CPSDN planning model and the solving algorithm. In order to fit the real application of integrated energy systems, two classic IEEE test systems with different scales are adopted in simulation, namely, IEEE 39-bus and IEEE LVNTS. The connectivity robustness and network efficiency are used as the performance metrics to evaluate the network planning algorithm.

4.1. Simulation with IEEE 39-Bus. Based on the power operational requirements, the initial CPS is established as Figure 3, following the IEEE 39-bus power system [24]. From the figure, we can find the heterogeneity of terminals in the CPSDN, and the types of terminals are shown in Table 2. Hence there is no radial feeder in this system, and it is not necessary to set the feeder terminal unit in the CPSDN. 
Input: $G,\{C\},\{M\}$

Output: Planned topology $T$

(1) Initialize the algorithm parameters: $M, N, c_{1}, c_{2}, w_{\max }, w_{\min }$; //Mis the Maximum number of Iterations, $N$ the Number of particles, $c_{1}$ and $c_{2}$ the learning factors and $w_{\max }$ and $w_{\min }$ the upper and lower bounds of weights.

(2) Initialize particles' position $x$ and flight speed $v ; / / x$ is the position vector and produced by $\{C\}$, who represents the possible communication link.

(3) Initialize P_best and G_best; //P_best is the optimal fitness value

(4) For $(i=0 ; i \leq M ; i++)$ among a particular particle and G_best is the global optimal value.

(5) $\quad$ For $(j=1 ; j \leq N ; j++)$

(6) Calculating inertia weight $w$ according to formula (7);

(7) Update particles' position $x_{i d}$ and flight speed $v_{i d}$ according to formula (6);

(8) If $\left(F\left(x_{i d}\right) \leq\right.$ P_best $)$

(9) Update P_best;

(10) End

(11) End

(12) If (P_best $\leq$ G_best)

(13) Update $T$ and G_best

(14) End

(15) End

(16) Output the planned topology $T$.

Algorithm 1: CPSND planning based on binary PSO algorithm.

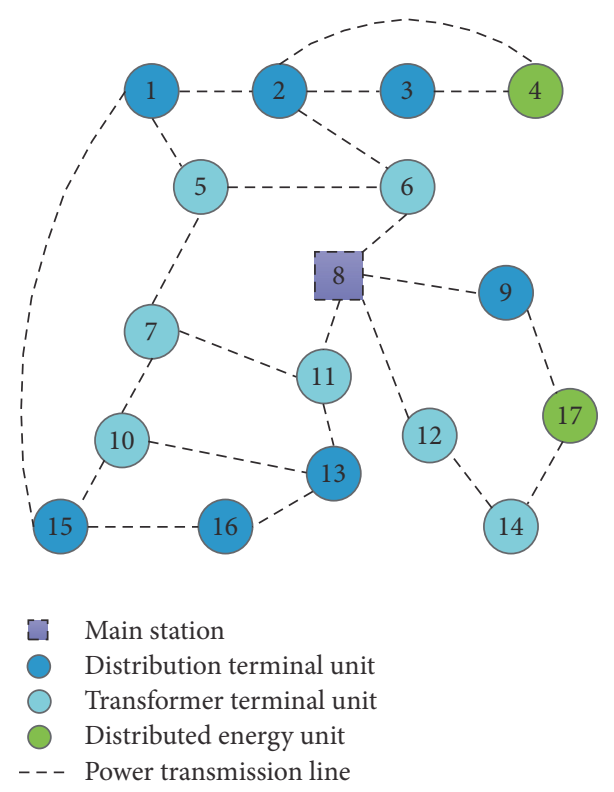

FIGURE 3: Initial CPS based on IEEE 39-bus power system.

To evaluate the algorithm's performance under different heterogeneous characteristics of CPSDN, we simulate four issues. From 1st to 4th program, the heterogeneous degree becomes deep, and the generalized node quality is shown in Table 3.

We use the improved binary PSO algorithm to solve the planning problem, which is realized with Matlab software. The characteristics and best parameters of the solver are obtained as shown in Table 4. Each result is the average value of at least 10 independent experiments to eliminate instability from the experimental distribution function. It is found that the binary PSO algorithm can converge to global optimization in 150 times of iteration, which shows that the proposed algorithm has better convergence speed.

Due to the large geographical distance, this paper uses the nodes randomly distributed in the plane of the $k * k$ size, where $k$ is the integer of the root of $n$ that equals 17. Adapting four programs of Table 2, our method can effectively balance the topological potential distribution shown in Figure 4 . Figure 4(a) uses the first node quality, and so forth. The 
TABLE 3: The generalized node quality of CPSDN's nodes.

\begin{tabular}{lccccc}
\hline Program & Master station & Distribution terminal unit & Transformer terminal unit & Feeder terminal unit & Distributed energy unit \\
\hline 1 & 0.2 & 0.2 & 0.2 & 0.2 & 0.2 \\
2 & 0.3 & 0.2 & 0.2 & 0.15 & 0.25 \\
3 & 0.45 & 0.15 & 0.13 & 0.12 & 0.15 \\
4 & 0.55 & 0.1 & 0.1 & 0.1 & 0.15 \\
\hline
\end{tabular}

TABLE 4: BPSO solver characteristics and parameters.

\begin{tabular}{lc}
\hline Type & Characters \\
\hline Num of iterations & $M=1000$ \\
Num of particles & $N=20$ \\
Learning factors & $c_{1}=c_{2}=2$ \\
Particle dimension & $D=17 * 17$ \\
Adaptive weight & $w_{\min }=0.4$ \\
\end{tabular}
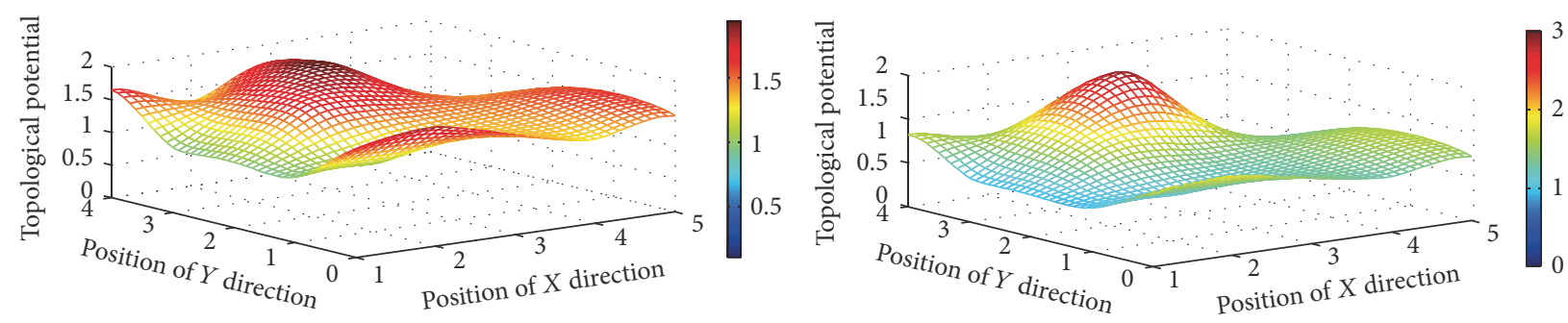

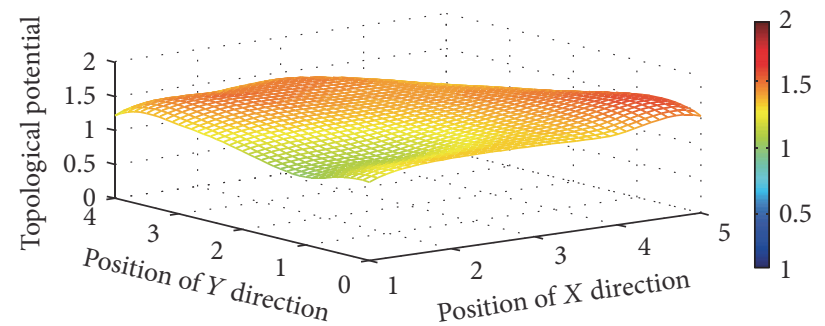

(a)
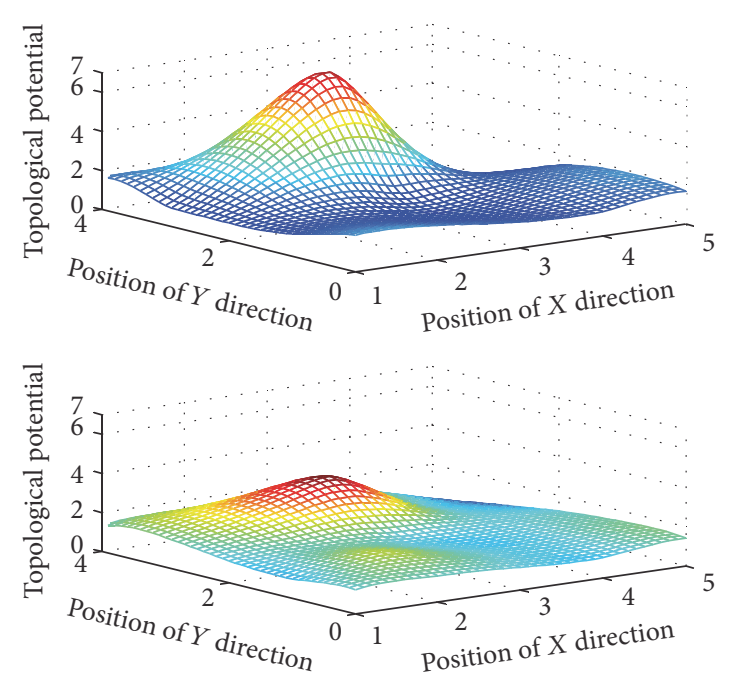

(c)

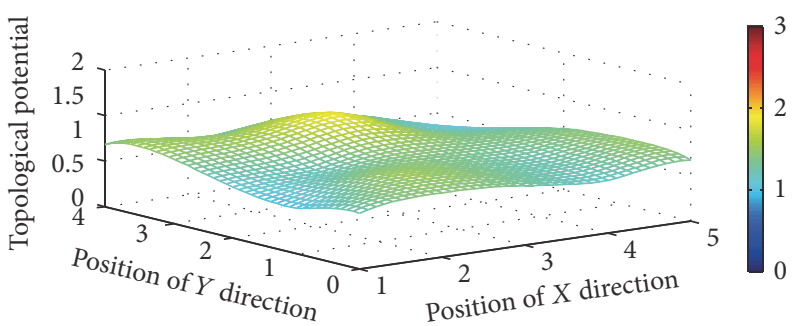

(b)

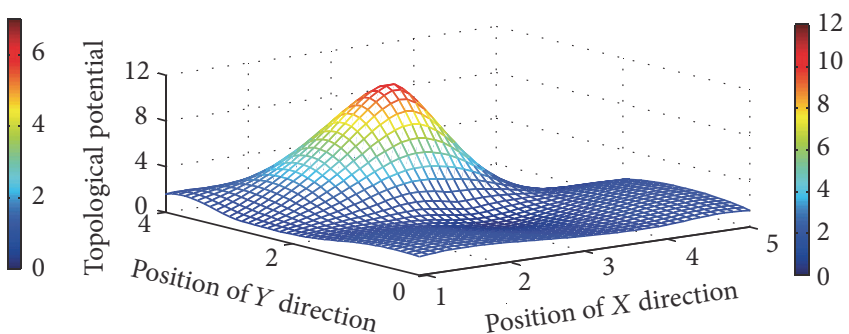

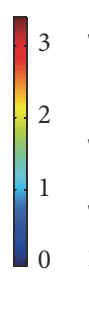

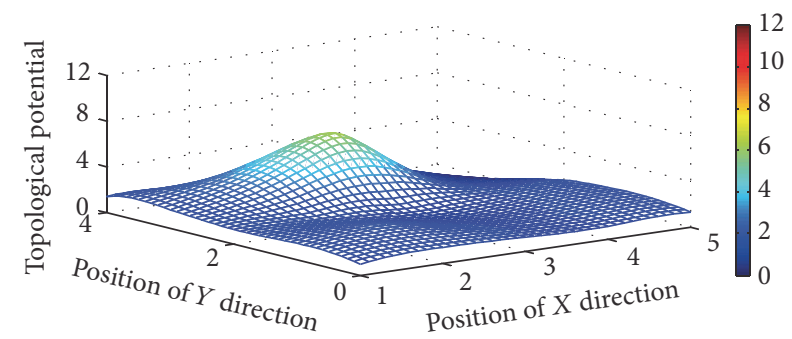

(d)

FIGURE 4: Comparison of the topological potential distribution between the initial network and the optimal ones. 


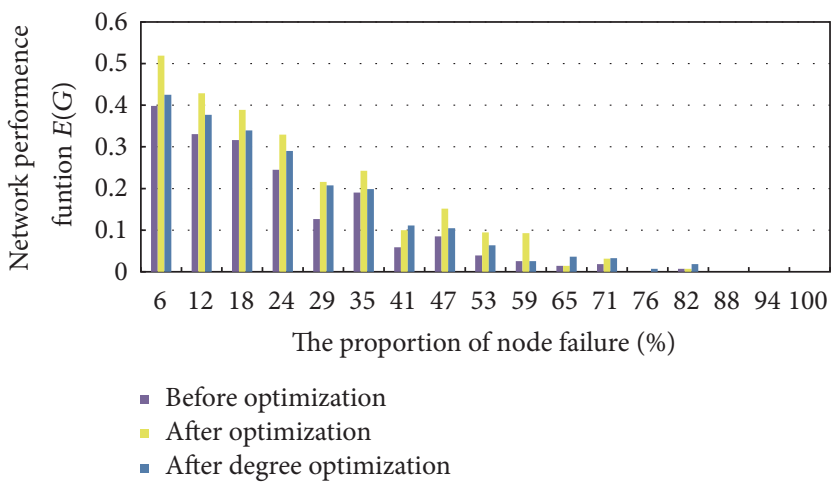

FIGURE 5: Network performance under the nodes random failure.

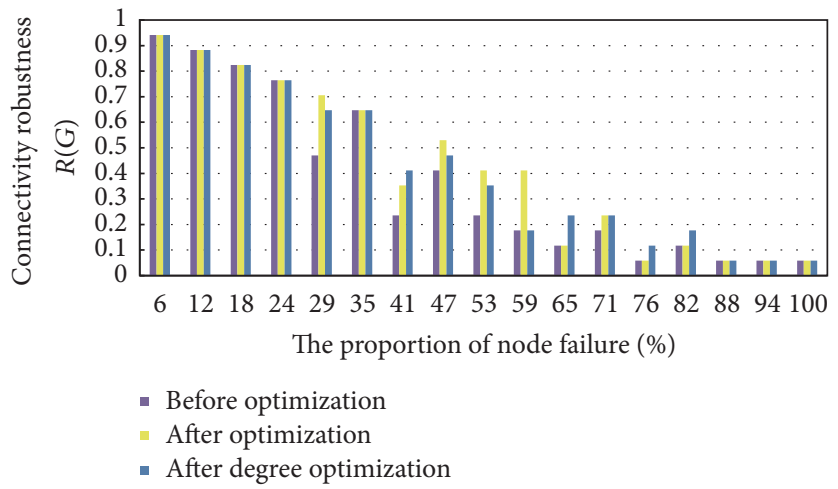

FIGURE 6: Connectivity robustness under the nodes random failure.

proposed method can well solve the planning problem of different heterogeneous networks.

Figures 4(a)-4(d) present the topological potential distribution with different degree of heterogeneity. The results show that in the initial CPS network, as the upper mesh plots in each figure, the distribution of topological potential is obvious, even if with high heterogeneity. The following shows the topological potential distribution of the optimal CPSDN topology with the proposed method in this paper, with which it is easy to find that the distribution of topological potential is more balanced.

In order to further illustrate the effectiveness of the proposed method, the connection degree [25] and the network performance function [26] are employed to compare the performance of the optimized network before and after. Therefore, the method of random failure of nodes is used to simulate the communication network in the case of largescale failure. Compared with the method of low degree node increasing links proposed by literature [11], the proposed method can improve the network performance, as shown in Figure 5.

The reliability of communication network is represented by the connectivity robustness. Using the random node failure method, the topology potential optimal algorithm can keep up with the higher reliability of the network, compared with the benchmark algorithm, shown in Figure 6.

4.2. Simulation of IEEE LVNTS. To evaluate the algorithm's performance for larger scale network, IEEE Low Voltage Network Test System (IEEE LVNTS) [27] is considered as another experiment example. IEEE LVNTS has many unique characters like numerous nodes, various load types, and so forth. The complete IEEE LVNTS is shown in Figure 7. In this paper, master station is deployed in the substation expressed by $\mathrm{P} 1$ in the figure. Transformers with deployed transformer terminal units are represented by the symbols P14, P16, and so forth. Distribution terminal units or distributed energy terminal units are hypothetically deployed in the spot network. Feeder terminals are deployed at the breakers considering the existence of long feeders.

Topology structure of larger scale network is optimized by the method of topological potential. The CPSDN of IEEE LVNTS system is modeled with the same generalized node quality in Figure 4(c). The effect of topological potential optimization is compared with the initial CPS network shown as Figure 8. The extent of topological potential equilibrium in Figure $8(\mathrm{~b})$ is even better than the previous example.

The method proposed in this paper can significantly improve the efficiency of the CPS communication network 


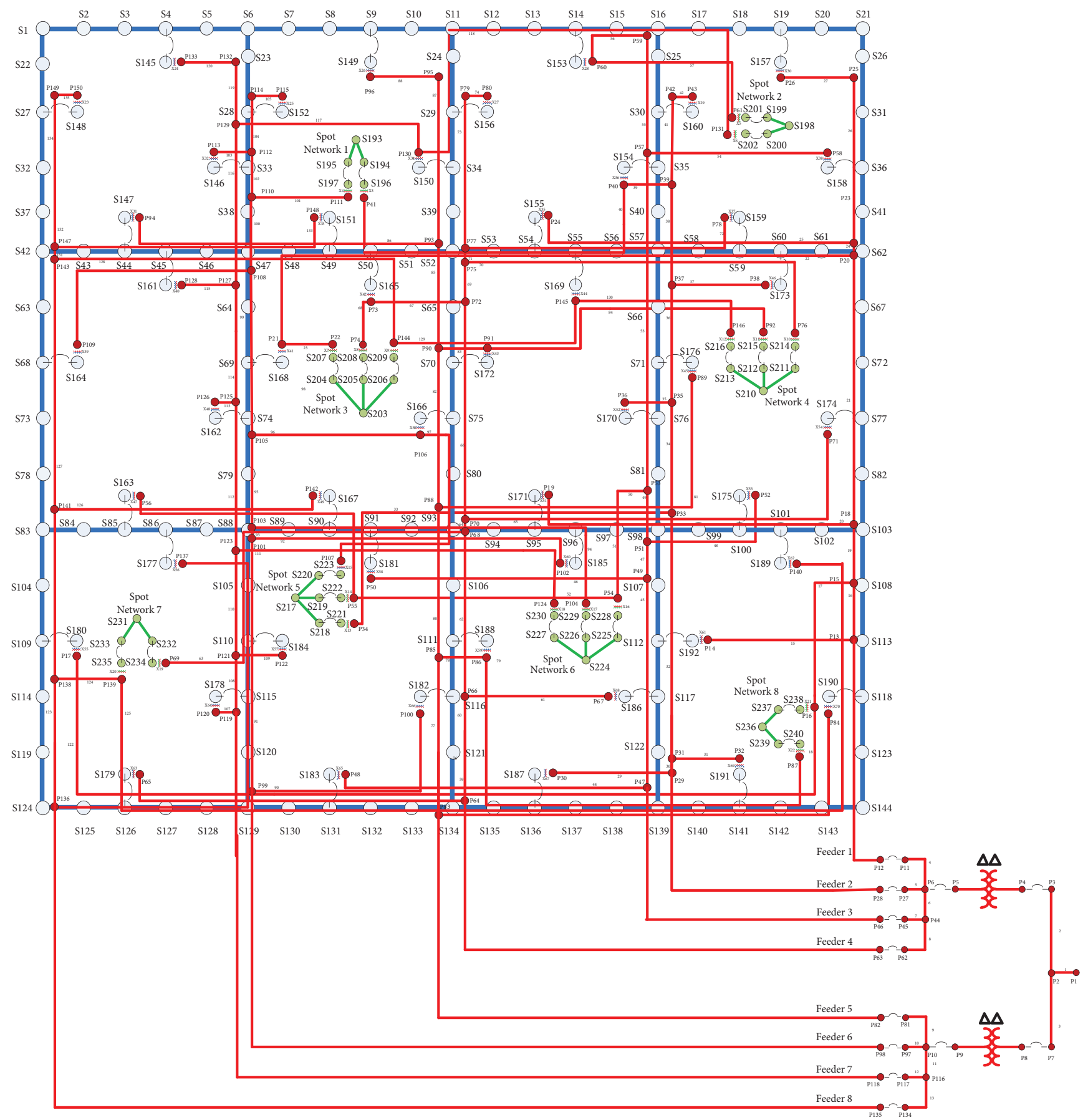

FIGURE 7: Complete IEEE LVNTS.

of the system shown as Figure 9. And this optimization can achieve better results than the degree optimization with all the node failure cycle. Without the limited number of added edges, the improvement of the network connectivity robustness is also more obvious compared with benchmark method as shown in Figure 10. Simulation results show that this method is also applicable to larger and more complex CPSDN.

\section{Conclusion}

The multisource heterogeneity of CPSDN has been discussed and a novel topological potential equilibrium optimal CSPDN planning model has been presented in this paper. With the generalized node quality, the CPS terminal's heterogeneity is formulated to characterize the degree of heterogeneity. An improved binary particle swarm optimization 


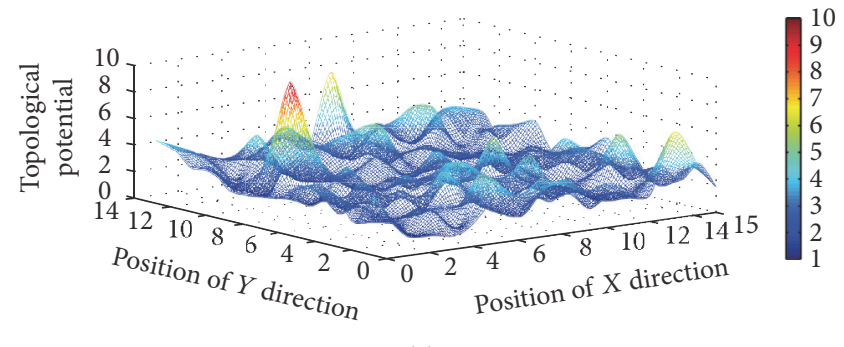

(a)

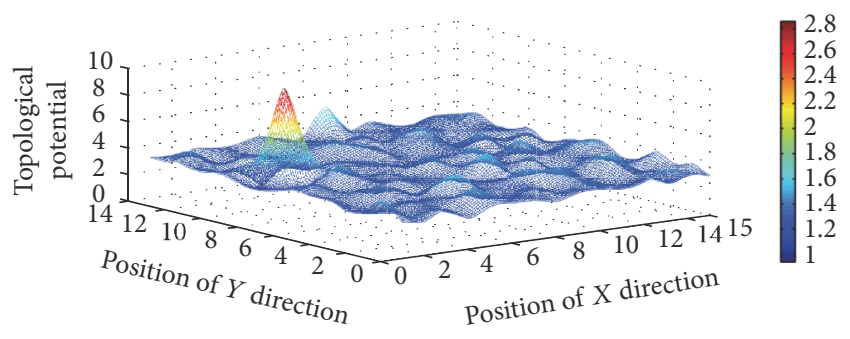

(b)

FIGURE 8: Comparison of the topological potential distribution between the initial network and the optimal one.

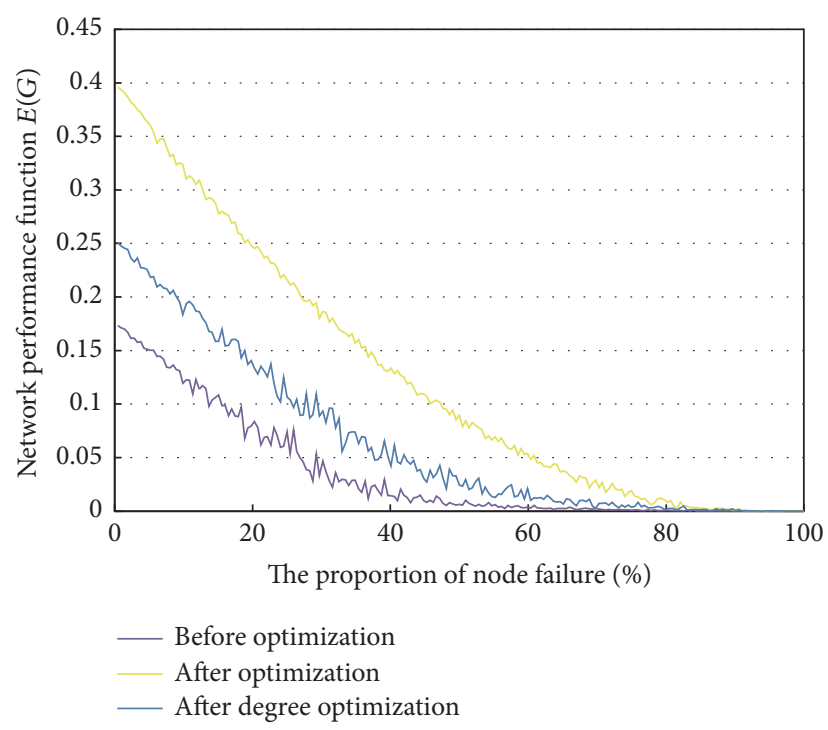

FIGURE 9: Network performance under the nodes random failure.

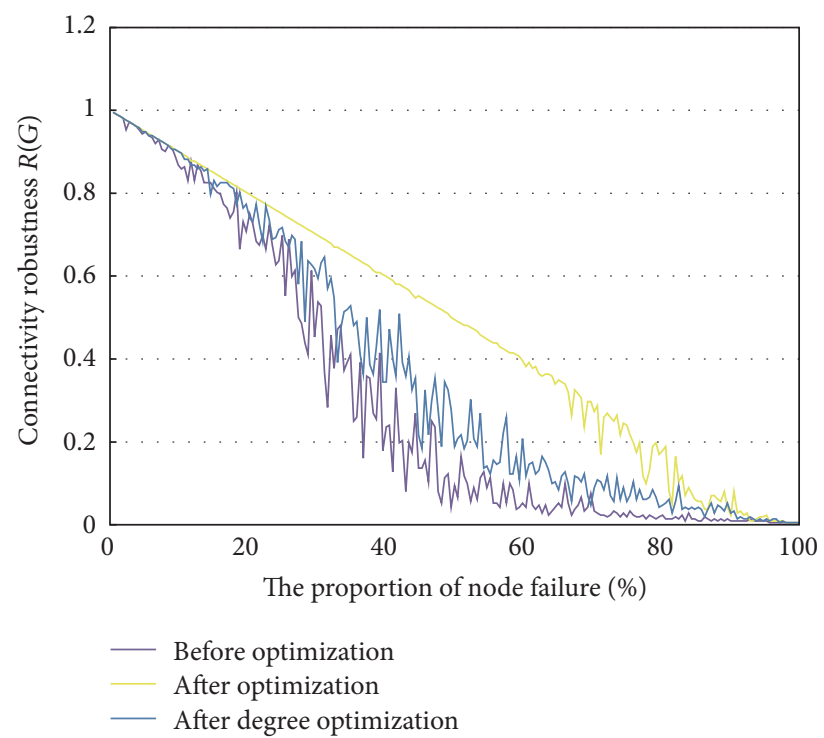

FIGURE 10: Connectivity robustness under the nodes random failure. algorithm is proposed to solve the complex planning scheme. The experimental results show that the proposed algorithm can greatly balance topological potential optimization and improve the reliability of CPSDN. This method can also be used in other heterogeneous network planning models, using different heterogeneity definition criteria.

\section{Competing Interests}

The authors declare that they have no competing interests.

\section{Acknowledgments}

This work was sponsored by the National High Technology Research and Development Program (863 Program) (2015AA050202), National Natural Science Foundation of China (61571324), and Natural Science Foundation of Tianjin (16JCZDJC30900).

\section{References}

[1] E. A. Lee, "Cyber-physical systems-are computing foundations adequate," in Position Paper for NSF Workshop on Cyber-Physical Systems: Research Motivation, Techniques and Roadmap, vol. 2, 2006.

[2] T. Sanislav and L. Miclea, "Cyber-physical systems-concept, challenges and research areas," Control Engineering and Applied Informatics, vol. 14, no. 2, pp. 28-33, 2012.

[3] S. Bu and F. R. Yu, "A game-theoretical scheme in the smart grid with demand-side management: towards a smart cyberphysical power infrastructure," IEEE Transactions on Emerging Topics in Computing, vol. 1, no. 1, pp. 22-32, 2013.

[4] S. Ahmad, B. Noor, and F. W. Karam, "Design and implementation of wireless sensor network for smart grids," International Journal of Advanced Computer Communication and Control, vol. 2, no. 1, 2016.

[5] S. Mohagheghi, J.-C. Tournier, J. Stoupis et al., "Applications of IEC 61850 in distribution automation," in Proceedings of the IEEE/PES Power Systems Conference and Exposition (PSCE '11), pp. 1-9, IEEE, Phoenix, Ariz, USA, March 2011.

[6] M. D. De Amorim, A. Ziviani, Y. Viniotis, and L. Tassiulas, "Practical aspects of mobility in wireless self-organizing networks [Guest Editorial]," IEEE Wireless Communications, vol. 15, no. 6, pp. 6-7, 2008. 
[7] A. A. Aziz, Y. A. Sekercioglu, P. Fitzpatrick, and M. Ivanovich, "A survey on distributed topology control techniques for extending the lifetime of battery powered wireless sensor networks," IEEE Communications Surveys and Tutorials, vol. 15, no. 1, pp. 121-144, 2013.

[8] H.-R. Liu, W.-X. Yin, T. Han, and M.-R. Dong, "Wireless sensor network fault tolerant topology for lifetime optimization," Acta Physica Sinica, vol. 63, no. 4, Article ID 040509, 2014.

[9] S. M. Jameii, K. Faez, and M. Dehghan, "Multiobjective optimization for topology and coverage control in wireless sensor networks," International Journal of Distributed Sensor Networks, vol. 2015, Article ID 363815, 11 pages, 2015.

[10] C. Sun, R.-R. Yin, X.-C. Hao, J.-J. Dou, and B. Liu, "Connected dominating set topology control algorithm of heterogeneous wireless sensor networks," Journal of Software, vol. 22, no. 9, pp. 2137-2148, 2011.

[11] J. Hu, J. Yu, J. Cao, M. Ni, and W. Yu, “Topological interactive analysis of power system and its communication module: a complex network approach," Physica A: Statistical Mechanics and Its Applications, vol. 416, pp. 99-111, 2014.

[12] Y. Wang, D. Liu, and Y. Lu, "Research on hybrid system modeling method of cyber physical system for power grid," Proceedings of the Chinese Society of Electrical Engineering, vol. 36, no. 6, pp. 1464-1470, 2016.

[13] Y. Yang, W. Pei, W. Deng, H. Xiao, and Z. Qi, "Optimal operation of grid-connected microgrid based on Benders decomposition," Electric Power Automation Equipment, vol. 34, no. 10, pp. 21-27, 2014.

[14] Z. Zhao and H. Hu, "A new service section based method to calculate service data flow of communication network for smart power distribution and utilization system," Power System Technology, vol. 35, no. 11, pp. 12-17, 2011.

[15] F. Xiao, Z. Zhang, and X. Yin, "An improved weighted IPACT algorithm of EPONs in smart distribution networks based on the requirements of relay protection," International Transactions on Electrical Energy Systems, vol. 26, no. 10, pp. 2107-2122, 2016.

[16] G. Han and B. Xu, "Modeling of intelligent distribution terminal according to IEC61850," Electric Power Automation Equipment, vol. 31, no. 2, pp. 104-107, 2011.

[17] D. Li, "Artificial intelligence with uncertainty," in Proceedings of the 4th International Conference onComputer and Information Technology (CIT '04), Wuhan, China, September 2004.

[18] J. Hu, Y. Han, and J. Hu, “Topological potential: modeling node importance with activity and local effect in complex networks," in Proceedings of the International Conference on Computer Modeling and Simulation (ICCMS '10), pp. 411-415, Sanya, China, January 2010.

[19] W.-Y. Gan, N. He, D.-Y. Li, and J.-M. Wang, "Community discovery method in networks based on topological potential," Journal of Software, vol. 20, no. 8, pp. 2241-2254, 2009.

[20] T. L. Saaty, "How to make a decision: the analytic hierarchy process," European Journal of Operational Research, vol. 48, no. 1, pp. 9-26, 1990.

[21] A. E. Jahromi and Z. B. Rad, "Optimal topological design of power communication networks using genetic algorithm," Scientia Iranica, vol. 20, no. 3, pp. 945-957, 2013.

[22] J. Kennedy, "Particle swarm optimization," in Encyclopedia of Machine Learning, pp. 760-766, Springer US, 2011.

[23] J.-H. Wen, K.-J. Zhong, L.-J. Tang et al., "Adaptive variableweighted support vector machine as optimized by particle swarm optimization algorithm with application of QSAR studies," Talanta, vol. 84, no. 1, pp. 13-18, 2011.
[24] X. Xiong, J. Tan, and X. Lin, "Routing algorithm for communication system in wide-area protection based on MPLS," Transactions of China Electrotechnical Society, vol. 28, no. 6, pp. 257-263, 2013.

[25] R. Albert and A.-L. Barabási, "Statistical mechanics of complex networks," Reviews of Modern Physics, vol. 74, no. 1, p. 47, 2002.

[26] J. Deng, "Structural reliability analysis for implicit performance function using radial basis function network," International Journal of Solids and Structures, vol. 43, no. 11-12, pp. 3255-3291, 2006.

[27] K. Schneider, P. Phanivong, and J. Lacroix, "IEEE 342-node low voltage networked test system," in Proceedings of the IEEE PES General Meeting, pp. 1-5, Washington, DC, USA, July 2014. 


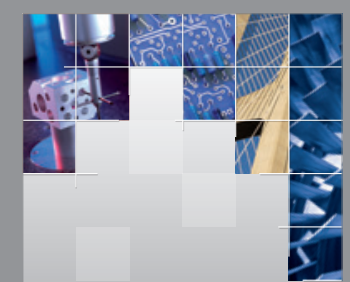

\section{Enfincering}
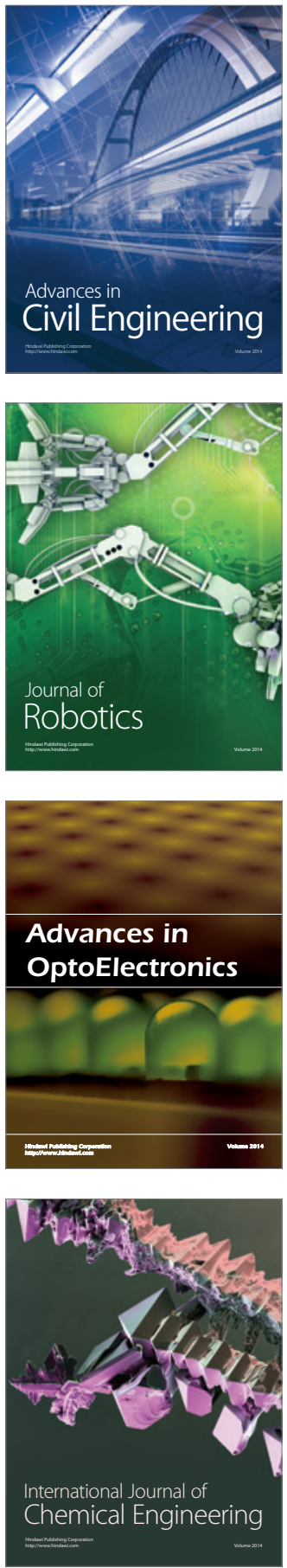

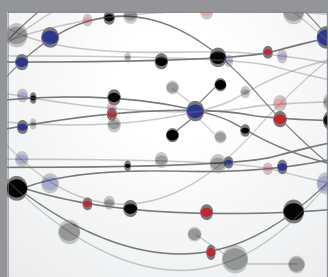

The Scientific World Journal

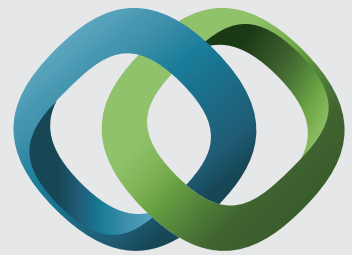

\section{Hindawi}

Submit your manuscripts at

https://www.hindawi.com
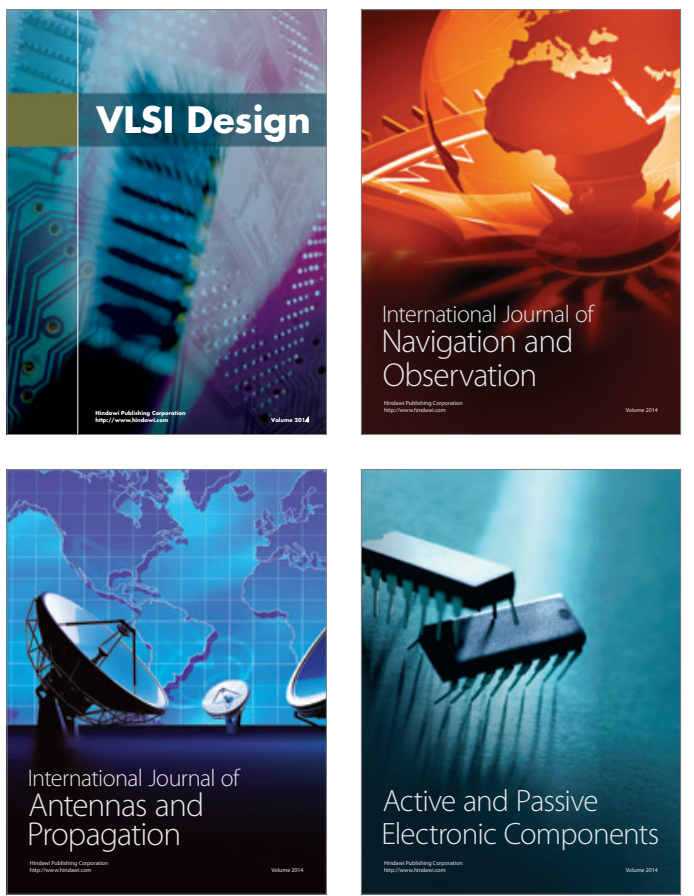
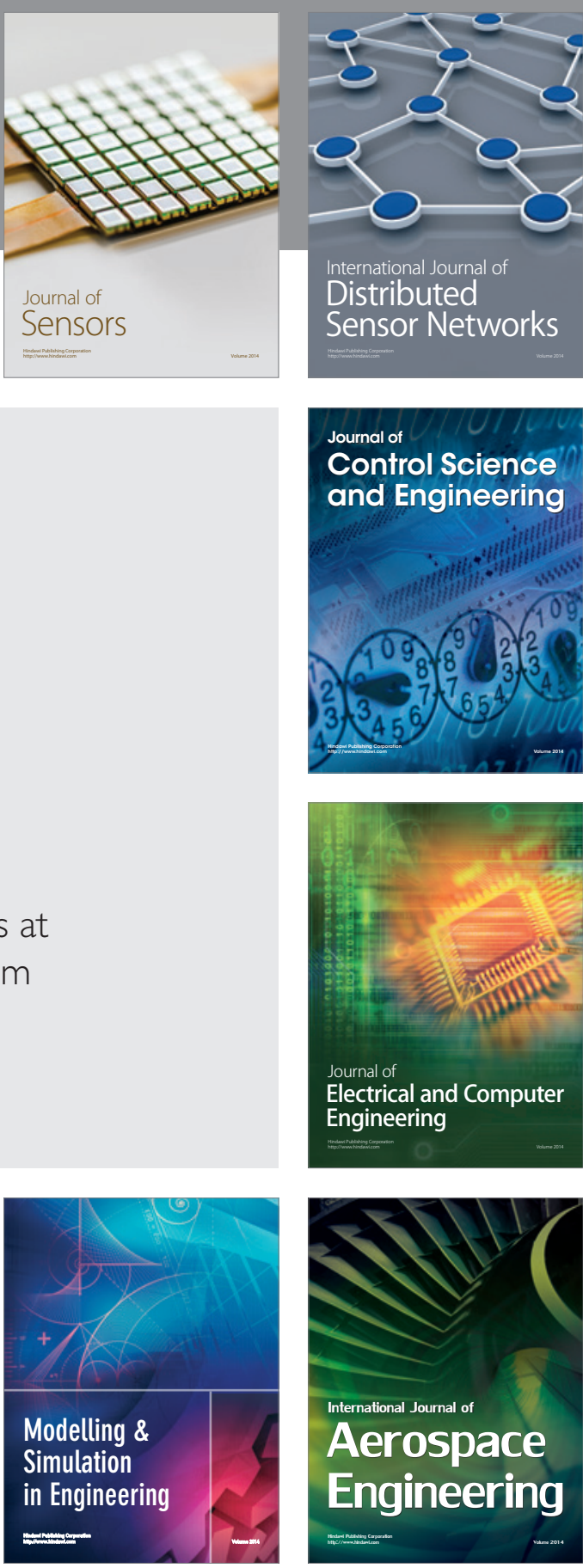

International Journal of

Distributed

Sensor Networks

$-$

Joumal of

Control Science

and Engineering
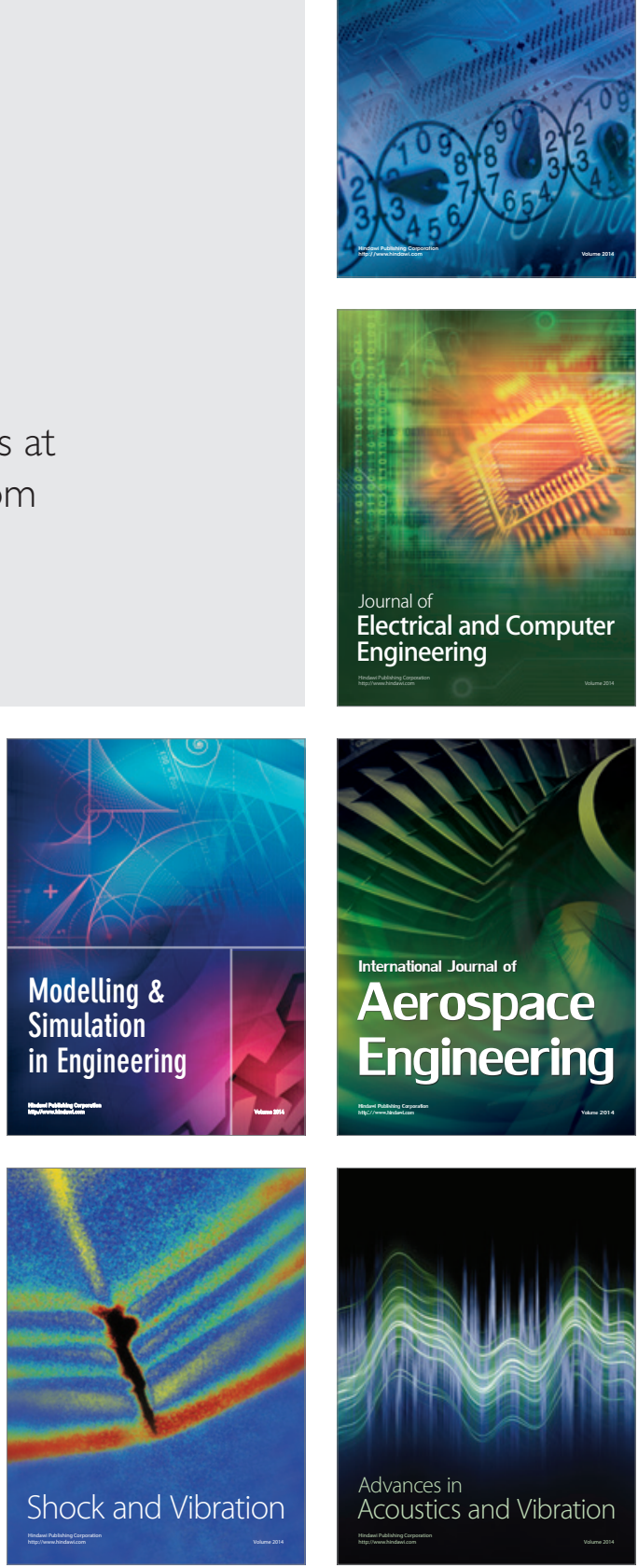